\title{
Association of candidate single nucleotide polymorphisms with somatic mutation of the epidermal growth factor receptor pathway
}

\author{
Samuel Wormald ${ }^{1,2^{*}}$, Liz Milla $a^{1,3}$ and Liam O'Connor ${ }^{1,2}$
}

\begin{abstract}
Background: Tumour growth in colorectal cancer and other solid cancers is frequently supported by activating mutations in the epidermal growth factor receptor (EGFR) signaling pathway (Patholog Res Int 2011:932932, 2011). Treatment of metastatic colorectal cancer with targeted anti-EGFR therapeutics such as cetuximab extends survival in only $25 \%$ of patients who test wild-type for KRAS, while the majority of patients prove resistant (J Clin Oncol 28(7):1254-1261, 2010).

Prediction of cetuximab responsiveness for KRAS wild-type colorectal cancers is currently not well defined, and prognostic biomarkers would help tailor treatment to individual patients. Somatic mutation of the EGFR signalling pathway is a prevalent mechanism of resistance to cetuximab (Nature 486(7404):532-536, 2012). If the human genome harbours variants that influence susceptibility of the EGFR pathway to oncogenic mutation, such variants could also be prognostic for cetuximab responsiveness.

Methods: We assessed whether patient genetic variants may associate with somatic mutation of the EGFR signalling pathway. We combined tumour mutation data from the Cancer Genome Atlas with matched patient genetic data, and tested for germline variants that associate with somatic mutation of the EGFR pathway (including EGFR, KRAS, BRAF, PTEN and PIK3CA).

Results: Two single nucleotide polymorphisms (SNPs) located $90 \mathrm{~kb}$ upstream of the TERT oncogene associated with somatic mutation of the EGFR pathway beyond the threshold of genome-wide significance: rs 7736074 $\left(P=4.64 \times 10^{-9}\right)$ and rs4975596 $\left(P=5.69 \times 10^{-9}\right)$. We show that allelic variants of rs7736074 and rs4975596 modulate TERT expression levels in multiple cancer types, and exhibit preliminary prognostic value for response to cetuximab.

Conclusions: We have identified two germline SNPs that associate with somatic mutation of the EGFR pathway, and may be prognostic for cetuximab responsiveness. These variants could potentially contribute to a panel of prognostic biomarkers for assessing whether metastatic colorectal cancer patients are likely to derive benefit from cetuximab treatment. Genotyping of a large cohort of cetuximab-treated colorectal cancer patients is called for to further clarify the association.
\end{abstract}

\footnotetext{
* Correspondence: wormald@wehi.edu.au

${ }^{1}$ Division of Systems Biology and Personalized Medicine, The Walter and Eliza

Hall Institute of Medical Research, Melbourne, Australia

${ }^{2}$ Department of Medical Biology, The University of Melbourne, Melbourne,

Australia

Full list of author information is available at the end of the article
} 


\section{Background}

The growth of solid tumours is frequently supported by aberrant expression of epidermal growth factor receptor
(EGFR) or activating mutations in downstream signalling components [1]. Monoclonal antibodies directed against EGFR, including cetuximab and panitumumab, have shown
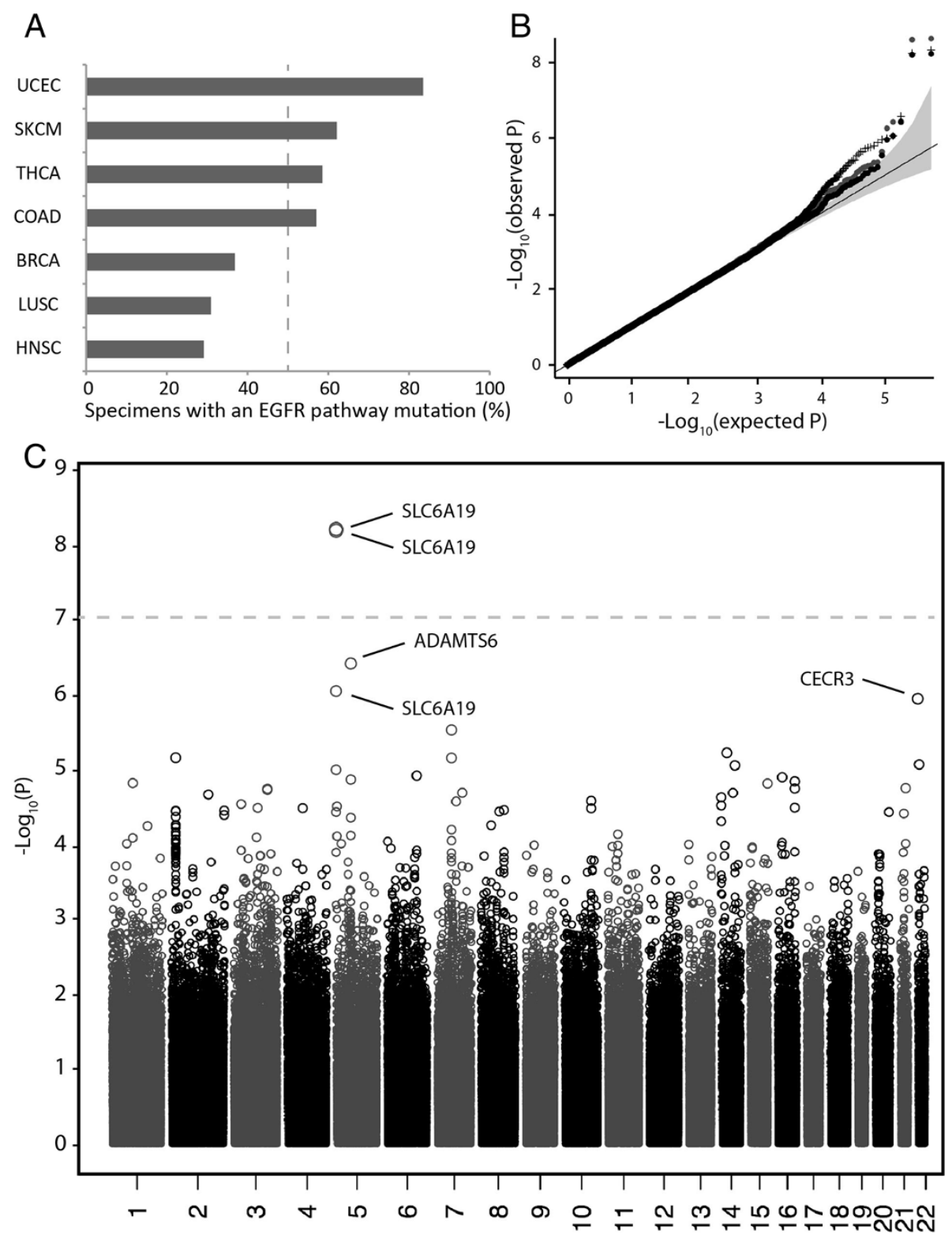

Chromosome

Figure 1 Frequencies of somatic mutation in the EGFR pathway for different solid tumour types. (A) TCGA tumour specimens were classified by mutation status for commonly mutated components of the EGFR pathway, including EGFR, KRAS, BRAF, PIK3CA and PTEN. The EGFR pathway was considered mutant if a non-synonymous mutation was detected in one or more of these factors. High and low mutation frequencies were determined by a 50\% cutoff (dashed line). Cancer types with little or no evidence of EGFR pathway mutation are not shown. Somatic mutations for individual specimens were obtained from publicly available TCGA somatic mutation data (based on MutSig analysis of whole-exome sequencing). LUAD was excluded from the study due to reported strong environmental association between KRAS mutation status and smoke exposure [17]. (B) Quantile-quantile plot of GWAS SNP p-values for association with somatic mutation status of the EGFR pathway. Cancer types included in the analysis were: UCEC, SKCM, THCA and COAD. Black points show p-values adjusted for population stratification using genomic inflation control; crosses show p-values adjusted for population stratification by incorporation of the top 10 eigenvectors as covariates; grey points show non-adjusted p-values. (C) Manhattan plot of GWAS SNP p-values for association with somatic mutation status of the EGFR pathway. P-values were adjusted for genomic inflation. Grey dashed line indicates the genomewide significance threshold $\left(p=8.78 \times 10^{-8}\right)$. Abbreviations: UCEC - uterine corpus endometrial carcinoma. SKCM - skin cutaneous melanoma. THCA thyroid carcinoma. COAD - colorectal adenocarcinoma. BRCA - breast invasive carcinoma. LUSC - lung squamous cell carcinoma. HNSC - head and neck squamous cell carcinoma. LUAD - lung adenocarcinoma. 
Table 1 Classification of TCGA patients according to EGFR pathway mutation status

\begin{tabular}{llll}
\hline & & \multicolumn{2}{l}{ Number of patients } \\
\cline { 3 - 4 } Cancer type & EGFRpath + & EGFRpath - & Total \\
\hline THCA & 187 & 135 & $\mathbf{3 2 2}$ \\
UCEC & 206 & 42 & $\mathbf{2 4 8}$ \\
SKCM & 131 & 88 & $\mathbf{2 1 9}$ \\
COAD/READ & 128 & 96 & $\mathbf{2 2 4}$ \\
All & 652 & 361 & $\mathbf{1 0 1 3}$ \\
\hline
\end{tabular}

Abbreviations: THCA thyroid carcinoma, UCEC uterine corpus endometrial carcinoma, SKCM skin cutaneous melanoma, COAD colorectal adenocarcinoma, READ rectal adenocarcinoma, EGFRpath + somatic mutation, EGFRpath - not mutated.

efficacy both as monotherapies and in combination with chemotherapy for the treatment of colorectal cancer (CRC) [2]. Despite providing new avenues of treatment for solid cancers, effectiveness in the clinic has proved variable. $40 \%$ of CRC cases harbor an activating mutation in KRAS and derive no benefit from anti-EGFR therapy, while only $13 \%$ of KRAS wild-type cases show an objective response $[3,4]$.

Regardless of their initial response, patients invariably develop resistance to targeted EGFR therapy [3,5,6]. Resistance is likely acquired by the emergence of mutations within EGFR or the EGFR pathway, including KRAS, BRAF, PIK3CA and PTEN. In KRAS wild-type CRC treated with cetuximab, 6 out of 10 cases acquire activating mutations in KRAS [3], and activating mutations in EGFR occur in 2 out of 10 cases [6]. Likewise, half of all non-small cell lung cancers treated with the EGFR inhibitors gefitinib or erlotinib acquire a second mutation in exon 20 of EGFR that confers resistance [5]. As response durations are typically measured in months, strategies to circumvent acquired drug resistance are needed.

The personalization of cancer care aims to predict effective therapy regimes according to the molecular profiles of individual patients and their cancers [7]. Germline SNPs in two components of the EGFR signalling pathway, EGF and Cyclin D1, are associated with overall survival in advanced CRC patients treated with cetuximab monotherapy [8], and a SNP in LIFR shows

Table 2 Top 5 SNPs identified by GWAS for EGFR pathway status

\begin{tabular}{llllll}
\hline Gene & SNP ID & Chromosome & Position $^{\mathbf{a}}$ & Region & P value $^{\mathbf{b}}$ \\
\hline SLC6A19 & rs7736074 & 5 & 1189456 & Upstream & $4.64 \times 10^{-9}$ \\
SLC6A19 & rs4975596 & 5 & 1189347 & Upstream & $5.69 \times 10^{-9}$ \\
ADAMTS6 & rs715676 & 5 & 64450473 & Intron & $1.56 \times 10^{-6}$ \\
SLC6A19 & rs6554634 & 5 & 1186121 & Upstream & $1.76 \times 10^{-6}$ \\
CECR3 & rs4819993 & 22 & 17761425 & Upstream & $3.77 \times 10^{-6}$ \\
\hline
\end{tabular}

${ }^{a}$ hg19 coordinates.

${ }^{\mathrm{b}}$ Adjusted for genomic inflation. association with response to cetuximab combination therapy [9]. At the tumour level, somatic mutations in EGFR, KRAS, BRAF, PTEN and PIK3CA are associated with poor response to anti-EGFR therapy in CRC $[2,10]$. Even the majority of cancers initially negative for these mutations fail to respond [2], probably because subpopulations harboring drug-resistant mutations have been selected [3]. The identification of germline biomarkers that can predict whether a cancer is predisposed to activating mutations in the EGFR pathway would therefore be an extremely useful therapeutic tool.

\section{Methods}

\section{Data sets}

Germline SNP data (Affymetrix SNP 6.0) for cancer patients were obtained from The Cancer Genome Atlas (TCGA - level 2 Birdseed output) [11]. Matched somatic mutation data and RNA seq data were obtained from the TCGA exome sequencing pipeline and the TCGA RNA seq pipeline respectively. Where multiple replicate specimens were available from a single patient, one representative specimen was selected at random. For association analysis, patients were only included where both germline SNP data and matched somatic mutation data were available. For RNA-seq analysis, patients were only included where both germline SNP data and matched tumour RNA-seq data were available.

Germline SNP chip data for Korean colorectal cancer patients and matched in vitro cetuximab response levels [9] were obtained from the Gene Expresison Omnibus [12] (GSE21228).

\section{Genome wide association analysis}

Genome wide SNP association was performed using the GWASTools package for R [13]. Associations were tested for using logistic regression under an additive model. For quality control, SNPs exhibiting $>5 \%$ missing genotype calls or non-Hardy-Weinberg equilibrium $(\mathrm{p}<0.001)$ were excluded. A relatively high minor allele frequency cutoff of $10 \%$ was chosen due to the moderate number of patients and the high frequency of the measured outcome within the cancer patient population (meaning that rare SNPs are unlikely to prove informative). Nonautosomal SNPs were also excluded. In total, 580,710 out of 906,600 SNPs on the Affymetrix Human SNP 6.0 array were included in the final analysis. The genomewide significance $\mathrm{p}$-value cutoff was calculated as 0.05 / $(580,710$ SNPs tested $)=8.61 \times 10^{-8}$. Measurement of the genomic inflation factor $(\lambda)$ and adjustment of $P$ values for genomic inflation was performed using the genomic control functionality of the METAL [14] software package. Alternatively, eigenvectors as determined by EIGENSTRAT [15] were included as covariates in a linear regression model. 

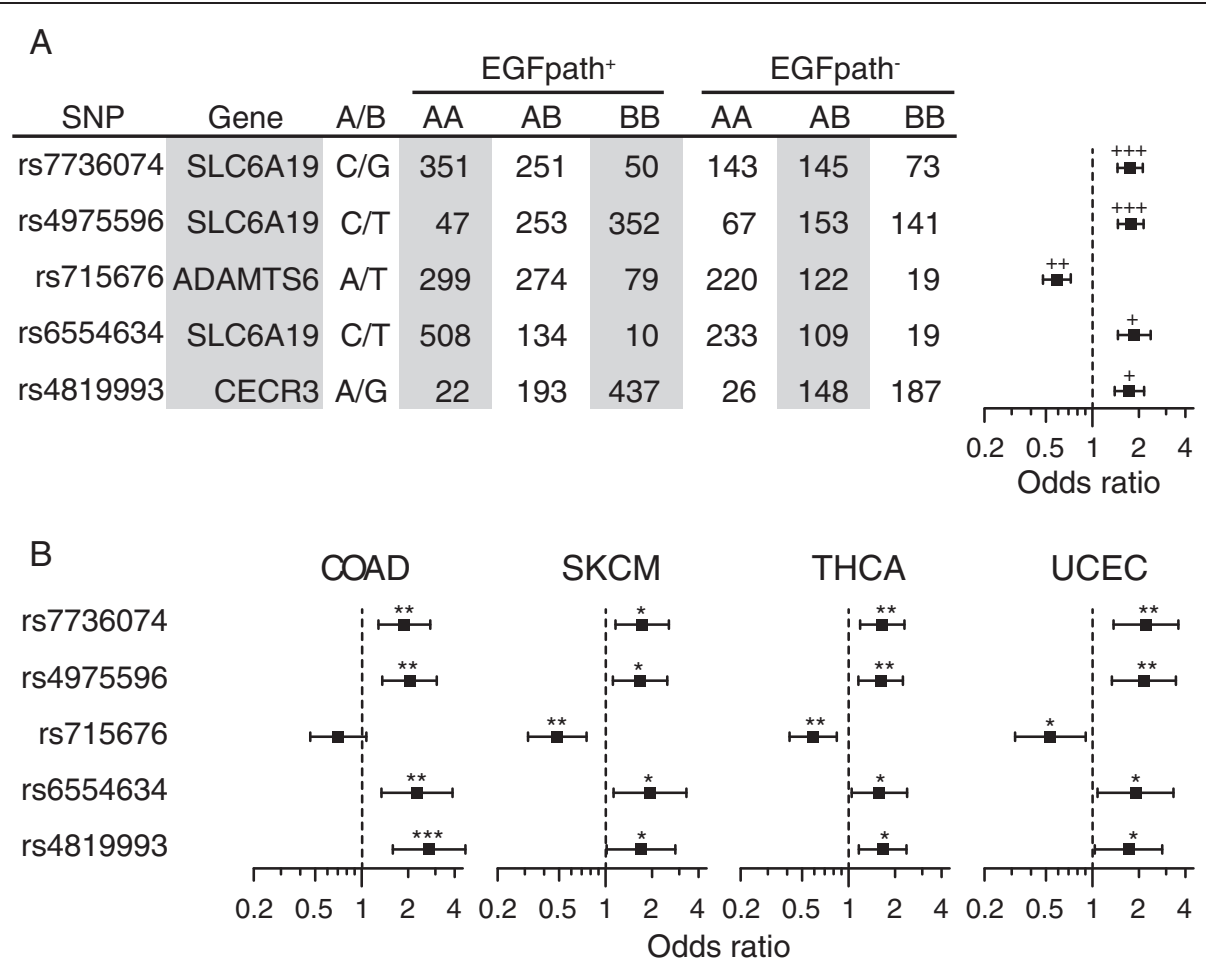

Figure 2 Patient genotype numbers and odds ratios for the top five SNPs associated with EGFR pathway status combined across four cancer types (COAD, SKCM, THCA and UCEC). (A) Odds ratios for combined analysis. (B) Odds ratios for individual cancer types. Error bars indicate 95\% confidence intervals. ${ }^{+++} \mathrm{P}<5 \times 10^{-9} ;{ }^{++} \mathrm{P}<5 \times 10^{-7} ;{ }^{+} \mathrm{P}<5 \times 10^{-6} ;{ }^{* * *} \mathrm{P}<5 \times 10^{-4} ;{ }^{* *} \mathrm{P}<5 \times 10^{-3} ;{ }^{*} \mathrm{P}<0.05$.

\section{RNA-Seq analysis of TERT expression levels}

Raw counts from TCGA RNA-seq data were processed using edgeR [16]. Briefly, counts were normalized within samples, and negative binomial linear models applied, allowing gene-level variance to be quantified using Cox-Reid estimates of common and tagwise dispersions. Differential expression was then tested for using a generalized linear model likelihood ratio test.

\section{Results}

\section{Genetic association with EGFR pathway status in cancer}

We sought to determine whether a patient's germline genetic profile influences susceptibility to mutation in EGFR or downstream signaling components. To approach this problem, we made use of The Cancer Genome Atlas (TCGA) [11] project which collects both somatic mutation data for patient tumours, as well as patients' germline genetic profiles. Individual cancer types within TCGA comprise too few patients to attempt large scale association analysis, however as somatic mutation of the EGFR pathway is a hallmark of multiple types of solid cancer types, we sought to maximize the power of our study by combining patients across multiple cancer types that exhibit high frequency of mutation in the EGFR pathway. We note this increase in patient numbers comes at the expense of potentially losing signals specific to only single cancer types.

First, somatic mutation frequencies for commonly mutated components of the EGFR pathway (EGFR, KRAS, BRAF, PIK3CA and PTEN) were assessed in multiple solid cancer types (Figure 1A). Cancer types with moderate to high frequencies of non-synonymous mutations in the EGFR pathway (> 50\%; Figure 1A) were combined for subsequent analysis: uterine corpus endometrial carcinoma (UCEC), skin cutaneous melanoma (SKCM), thyroid carcinoma (THCA) and colorectal adenocarcinoma (COAD). As each of these cancer types exhibits dependency on components of the EGFR pathway at similar frequencies (Figure 1A), we anticipated that through combining them we might uncover a common genetic predisposition.

Next, a genome-wide association study (GWAS) was performed to measure germline genetic association with somatic mutation of the EGFR pathway. Combining cases of UCEC, SKCM, THCA and COAD yielded a total of 1,013 patients (Table 1). From 569,429 SNPs across 1,013 germline samples, a low genomic inflation factor of 1.026 was measured, suggesting a minimal level of population stratification (Figure 1B).

Two SNPs were detected beyond the genome-wide significance threshold $\mathrm{P}<8.78 \times 10^{-8}$ : rs7736074 $(\mathrm{P}=4.64 \times$ 


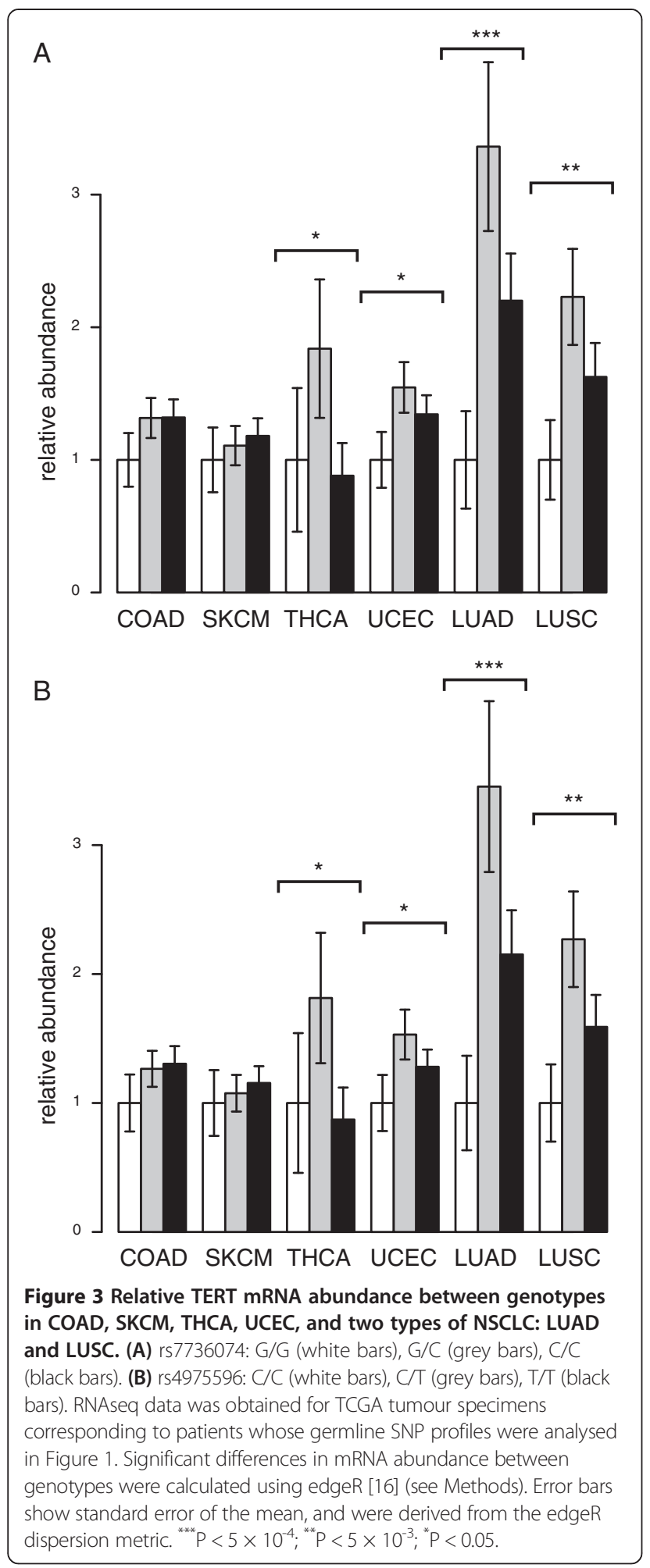

$\left.10^{-9}\right)$ and rs4975596 ( $\mathrm{P}=5.69 \times 10^{-9}$; see Figure $1 \mathrm{C}$, Table 2$)$. These SNPs, located on the short arm of chromosome 5 and separated by $109 \mathrm{bp}$, show a high degree of linkage disequilibrium, with $R^{2}=1.0$ for individuals of northernEuropean ancestry, and $\mathrm{R}^{2}=0.935$ for individuals of
Chinese or Japanese ancestry [18]. The close linkage between rs7736074 and rs4975596 provide important validation that the observed association is not a technical artifact of the SNP genotyping platform.

An additional quality control measure, principle component analysis, indicated that mutation status of the EGFR pathway is not simply driven by population structure (Additional file 1: Figure S1). Furthermore, different approaches of accounting for population structure did not dramatically alter the p-values for rs7736074 and rs4975596 (Figure 1B and Additional file 1: Figure S2). At the probe level, genotype intensity groups are generally well defined (Additional file 1: Figure S3), however we identified some samples with genotype call p-values above 0.05 ( $4 \%$ for rs $7736074,7 \%$ for rs 4975596 ), indicating lower confidence calls (Additional file 1: Figure S4). Both SNPs remain beyond genome-wide significance with these lower-confidence calls excluded from the analysis (rs7736074: 9.46×10-9; rs4975596: $2.67 \times 10^{-8}$ ).

Rs7736074 and rs4975596 are located approximately $12 \mathrm{~kb}$ upstream of SLC6A19, and $90 \mathrm{~kb}$ downstream of the gene encoding telomerase reverse transcriptase (TERT). Genetic variants near TERT are strongly associated with predisposition to eight or more different cancer types [19], suggesting a potential mechanism by which rs7736074 and rs4975596 could influence the oncogenic potential of the EGFR signaling pathway through modulation of TERT activity.

We also identified three additional SNPs that appear suggestive based on visual inspection of the quantile distribution for SNP P values, despite failing to achieve genome-wide significance (Figure 1B). Of the top five SNPs identified, four were located on chromosome 5, and one on chromosome 22 (Table 2). Uniform effect sizes were observed both for the combined analysis (Figure 2A) and for individual cancer types (Figure 2B).

\section{SNPs rs7736074 and rs4975596 associate with TERT expression levels}

The 15p5.33 locus, harboring SLC6A19 and TERT, is of particular importance in non-small cell lung cancer (NSCLC), where copy number amplification of the region is found in $78 \%$ of cases [20]. As SNPs at this locus could potentially influence oncogenesis by modulating TERT expression, we examined whether rs7736074 and rs4975596 associate with TERT expression levels in COAD, SKCM, THCA, UCEC, as well as for two subtypes of NSCLC (squamous cell or adenocarcinoma). We detected modest significant differences $(\mathrm{P}<0.05)$ in TERT expression between genotypes for THCA and UCEC, and larger significant differences for the two NSCLC subtypes $(\mathrm{P}<0.005$ and $\mathrm{P}<0.0005$; Figure $3 \mathrm{~A}$ and $3 \mathrm{~B}$ ). We observed similar trends for COAD and SKCM, however these effects were not significant. We hypothesize that the observed 
differences between cancer types may be attributable to differing degrees of cellular heterogeneity, or to differing degrees of TERT sensitivity.

TERT expression profiles for rs7736074 and rs4975596 were nearly identical, reflecting the high degree of linkage between these polymorphisms (Figure $3 \mathrm{~A}$ and $3 \mathrm{~B}$ ). The relationship between genotype and TERT expression was generally consistent between cancer tumour types (Figure 3A and 3B). Heterozygotes typically exhibited heightened expression levels, suggesting a complex relationship between genotype and other factors (such as copy-number or methylation) in determining TERT expression levels. In particular, the substantial differential expression of TERT between genotypes in the two NSCLC subtypes suggests that genotype could play a role in determining copy-number amplification of TERT.

\section{SNPs rs7736074 and rs4975596 associate with in vitro tumor sensitivity to cetuximab}

Understanding how germline genetic variation influences the EGFR pathway in cancer may aid in prediction of patient responses to targeted therapeutics. To test this hypothesis, and verify our GWAS findings in an independent population, we examined the association of SNPs from Table 2 with in vitro tumor response to cetuximab (Erbitux) using publicly available SNP data for 118 Korean colorectal cancer patients [9] (GEO series GSE21228; association with Erbitux response in the absence of chemotherapy). The profile of odds ratios for in vitro response to cetuximab (as measured by tumor cell viability) was concordant with the odds ratios observed for somatic mutation of the EGFR pathway (Figure 4; compare with Figure 2B). Significant association with in vitro cetuximab response was observed for SLC6A19 SNPs rs7736074 and rs4975596 ( $\mathrm{P}=0.003$ and 0.002 respectively). This finding supports our hypothesis that SNPs predictive of EGFR pathway mutation status may serve as informative biomarkers for predicting cetuximab response.

\section{Discussion}

At the molecular level, most human cancers can be classified into one or more subtypes of disease. The germline genetic profile of a patient can influence predisposition to specific cancer subtypes; in breast cancer, for example, FGFR2 variants are strongly associated with ER-positive but not ER-negative breast cancer [21]. In CRC, outgrowth of tumour subpopulations harboring mutations in components of the EGFR pathway is strongly associated with acquired resistance to cetuximab [4]. Cancer heterogeneity may confound the detection of such mutations by biopsy, or they may arise during the course of treatment. This study aimed to determine whether specific germline genetic factors may predispose patients to the acquisition of mutations in the EGFR pathway, and thus to cetuximab resistance. By including multiple components of the EGFR pathway in our association analysis, we aimed to isolate genetic variants that influence the EGFR pathway as a whole, as we reasoned these would likely be most informative.

We identified germline SNPs at 15p5.33 that associate with somatic mutation of the EGFR signaling pathway in TCGA patients. In an attempt to further validate this finding, we examined association of the SNPs with in vitro resistance to cetuximab (which likely reflects to some extent the mutation status of the EGFR pathway) in an independent cohort of CRC patients, and found them to be significant.

15 p5.33 is a hotspot of genetic predisposition for multiple cancer types, probably because oncogenesis and cell immortalization are closely linked with the telomere maintenance activities of TERT [19]. We postulate that the SNPs we identified may be in linkage with a regulatory element that modulates TERT expression. Consistent with this hypothesis, we found TERT mRNA

\begin{tabular}{|c|c|c|c|c|c|c|c|c|}
\hline \multirow[b]{3}{*}{ SNP } & \multirow[b]{3}{*}{ Gene } & \multicolumn{6}{|c|}{ Cetuximab responder status } & \\
\hline & & \multicolumn{3}{|c|}{ Non-responsive } & \multicolumn{3}{|c|}{ Responsive } & \\
\hline & & AA & $A B$ & $\mathrm{BB}$ & AA & $A B$ & $\overline{\mathrm{BB}}$ & \\
\hline rs7736074 & SLC6A19 & 37 & 36 & 14 & 6 & 13 & 12 & $\stackrel{* *}{\longrightarrow}$ \\
\hline rs4975596 & SLC6A19 & 14 & 34 & 39 & 12 & 13 & 6 & $\stackrel{*}{\longrightarrow}$ \\
\hline rs715676 & ADAMTS6 & 21 & 45 & 21 & 5 & 16 & 10 & - \\
\hline rs6554634 & SLC6A19 & 65 & 17 & 5 & 20 & 8 & 2 & \\
\hline rs4819993 & CECR3 & 10 & 34 & 41 & 5 & 14 & 12 & 一 \\
\hline
\end{tabular}

Figure 4 SNPs rs4975596 and rs7736074 associate with in vitro tumour sensitivity to cetuximab. Patient genotypes and odds ratios were calculated for in vitro response to cetuximab, using the same panel of SNPs as in Figure 2. Genotype and response data were obtained for 118 Korean colorectal cancer patients from the GEO database (accession GEO 21228) as described previously [9]. 
expression levels to be associated with genotype at rs4975596/rs7736074 in multiple cancer types. Association was strongest in squamous-cell carcinomas and adenocarcinomas of the lung, where the 15p5.33/TERT locus is amplified at particularly high frequency [20]. The other cancer types we examined exhibited similar regulatory trends albeit at decreased magnitude and significance, possibly due to differences in TERT dependence, tumour heterogeneity, or the action of alternative regulatory pathways at rs4975596/rs7736074 in lung cancer.

Numerous studies have reported regulation of TERT by EGFR-responsive factors including Wnt/B-catenin [22], Myc [23], and NFkB [24]. Further evidence for a regulatory link between EGFR and TERT was reported recently in malignant glioma, where $92 \%$ of cases harboring EGFR amplification were accompanied by a mutation in the TERT promoter [25]. Polymorphisms that disrupt a regulatory element linking EGFR signaling to TERT expression would thus impede the oncogenic potential of the EGFR pathway, and may reduce the likelihood of the pathway succumbing to somatic mutation.

\section{Conclusion}

The EGFR pathway induces pro-proliferative and antiapoptotic signals, and constitutes a convenient target for somatic mutation in cancer. The occurrence of such a mutation can impede the effectiveness of anti-EGFR therapeutics such as cetuximab. We used TCGA patient data to assess whether genetic variants may predispose to somatic mutation of the EGFR pathway. We identified two SNPs located $90 \mathrm{~kb}$ upstream of TERT, rs7736074 and rs4975596, that associate with EGFR pathway mutation $\left(\mathrm{P}<=5.69 \times 10^{-9}\right)$. We found the same two SNPs were also predictive of in vitro cetuximab resistance using publicly available genetic data from Korean colorectal cancer patients [9]. Our results suggest that genetic variants may predispose to somatic mutation of the EGFR pathway, and consequently to resistance with anti-EGFR therapeutics. Larger studies are called for to further characterize the contribution of patient genetic variation to anti-EGFR therapeutic resistance.

\section{Additional file}

Additional file 1: This file comprises Supplementary Figures S1-S4.

\section{Competing interests}

The authors declare they have no competing interests.

\section{Authors' contributions}

SW designed the study, designed and performed the analysis, analysed results, and prepared the manuscript. LM processed data, assisted with the analysis, and helped write the manuscript. LO assisted with the analysis, discussed results, and helped write the manuscript. All authors read and approved the final manuscript.

\section{Acknowledgements}

S.W. is supported by an NHMRC Biomedical Postdoctoral Fellowship (519795). This research was supported by the VLSCl's Life Sciences Computation Centre, a collaboration between Melbourne, Monash and La Trobe Universities and an initiative of the Victorian Government, Australia.

\section{Author details}

'Division of Systems Biology and Personalized Medicine, The Walter and Eliza Hall Institute of Medical Research, Melbourne, Australia. ${ }^{2}$ Department of Medical Biology, The University of Melbourne, Melbourne, Australia. ${ }^{3} \mathrm{VLSCl}$ Life Sciences Computation Centre, The University of Melbourne, Melbourne, Australia.

Received: 12 July 2013 Accepted: 21 October 2013

Published: 23 October 2013

\section{References}

1. Krasinskas AM: EGFR signaling in colorectal carcinoma. Patholog Res Int 2011, 2011:932932.

2. Bardelli A, Siena S: Molecular mechanisms of resistance to cetuximab and panitumumab in colorectal cancer. J Clin Oncol 2010, 28(7):1254-1261.

3. Misale S, Yaeger R, Hobor S, Scala E, Janakiraman M, Liska D, Valtorta E, Schiavo R, Buscarino M, Siravegna G, et al: Emergence of KRAS mutations and acquired resistance to anti-EGFR therapy in colorectal cancer. Nature 2012, 486(7404):532-536.

4. Karapetis CS, Khambata-Ford S, Jonker DJ, O'Callaghan CJ, Tu D, Tebbutt NC, Simes RJ, Chalchal H, Shapiro JD, Robitaille S, et al: K-ras mutations and benefit from cetuximab in advanced colorectal cancer. $N$ Engl I Med 2008, 359(17):1757-1765.

5. Kobayashi S, Boggon TJ, Dayaram T, Janne PA, Kocher O, Meyerson M, Johnson BE, Eck MJ, Tenen DG, Halmos B: EGFR mutation and resistance of non-small-cell lung cancer to gefitinib. N Engl J Med 2005, 352(8):786-792.

6. Montagut C, Dalmases A, Bellosillo B, Crespo M, Pairet S, Iglesias M, Salido M, Gallen M, Marsters S, Tsai SP, et al: Identification of a mutation in the extracellular domain of the epidermal growth factor receptor conferring cetuximab resistance in colorectal cancer. Nat Med 2012, 18(2):221-223.

7. Chan IS, Ginsburg GS: Personalized medicine: progress and promise. Annu Rev Genomics Hum Genet 2011, 12:217-244.

8. Zhang W, Gordon M, Press OA, Rhodes K, Vallbohmer D, Yang DY, Park D, Fazzone W, Schultheis A, Sherrod AE, et al: Cyclin D1 and epidermal growth factor polymorphisms associated with survival in patients with advanced colorectal cancer treated with Cetuximab. Pharmacogenet Genomics 2006, 16(7):475-483.

9. Kim JC, Kim SY, Cho DH, Ha YJ, Choi EY, Kim CW, Roh SA, Kim TW, Ju H, Kim YS: Novel chemosensitive single-nucleotide polymorphism markers to targeted regimens in metastatic colorectal cancer. Clin Cancer Res 2011, 17(5):1200-1209.

10. De Roock W, De Vriendt V, Normanno N, Ciardiello F, Tejpar S: KRAS, BRAF, PIK3CA, and PTEN mutations: implications for targeted therapies in metastatic colorectal cancer. Lancet Oncol 2011, 12(6):594-603.

11. The Cancer Genome Atlas. http://cancergenome.nih.gov/.

12. Gene Expression Omnibus. www.ncbi.nlm.nih.gov/geo/.

13. Gogarten SM, Bhangale T, Conomos MP, Laurie CA, McHugh CP, Painter I, Zheng X, Crosslin DR, Levine D, Lumley T, et al: GWASTools: an R/Bioconductor package for quality control and analysis of genomewide association studies. Bioinformatics 2012, 28(24):3329-3331.

14. Willer CJ, Li Y, Abecasis GR: METAL: fast and efficient meta-analysis of genomewide association scans. Bioinformatics 2010, 26(17):2190-2191.

15. Price AL, Patterson NJ, Plenge RM, Weinblatt ME, Shadick NA, Reich D: Principal components analysis corrects for stratification in genome-wide association studies. Nat Genet 2006, 38(8):904-909.

16. Robinson MD, McCarthy DJ, Smyth GK: EdgeR: a Bioconductor package for differential expression analysis of digital gene expression data. Bioinformatics 2010, 26(1):139-140.

17. Tam IY, Chung LP, Suen WS, Wang E, Wong MC, Ho KK, Lam WK, Chiu SW, Girard L, Minna JD, et al: Distinct epidermal growth factor receptor and KRAS mutation patterns in non-small cell lung cancer patients with different tobacco exposure and clinicopathologic features. Clin Cancer Res 2006, 12(5):1647-1653. 
18. Johnson AD, Handsaker RE, Pulit SL, Nizzari MM, O'Donnell CJ, de Bakker PI: SNAP: a web-based tool for identification and annotation of proxy SNPs using HapMap. Bioinformatics 2008, 24(24):2938-2939.

19. Baird DM: Variation at the TERT locus and predisposition for cancer. Expert Rev Mol Med 2010, 12:e16.

20. Kang JU, Koo SH, Kwon KC, Park JW, Kim JM: Gain at chromosomal region 5p15.33, containing TERT, is the most frequent genetic event in early stages of non-small cell lung cancer. Cancer Genet Cytogenet 2008, 182(1):1-11.

21. Garcia-Closas M, Hall P, Nevanlinna H, Pooley K, Morrison J, Richesson DA, Bojesen SE, Nordestgaard BG, Axelsson CK, Arias JI, et al: Heterogeneity of breast cancer associations with five susceptibility loci by clinical and pathological characteristics. PLoS Genet 2008, 4(4):e1000054.

22. Zhang $Y$, Toh $L$, Lau $P$, Wang $X$ : Human telomerase reverse transcriptase ( $\mathrm{hTERT}$ ) is a novel target of the Wnt/beta-catenin pathway in human cancer. J Biol Chem 2012, 287(39):32494-32511.

23. Wu KJ, Grandori C, Amacker M, Simon-Vermot N, Polack A, Lingner J, Dalla-Favera R: Direct activation of TERT transcription by c-MYC. Nat Genet 1999, 21(2):220-224.

24. Yin L, Hubbard AK, Giardina C: NF-kappa B regulates transcription of the mouse telomerase catalytic subunit. J Biol Chem 2000, 275(47):36671-36675.

25. Arita H, Narita Y, Fukushima S, Tateishi K, Matsushita Y, Yoshida A, Miyakita Y, Ohno M, Collins VP, Kawahara N, et al: Upregulating mutations in the TERT promoter commonly occur in adult malignant gliomas and are strongly associated with total 1p19q loss. Acta Neuropathol 2013.

doi:10.1186/1755-8794-6-43

Cite this article as: Wormald et al:: Association of candidate single nucleotide polymorphisms with somatic mutation of the epidermal growth factor receptor pathway. BMC Medical Genomics 2013 6:43.

\section{Submit your next manuscript to BioMed Central and take full advantage of:}

- Convenient online submission

- Thorough peer review

- No space constraints or color figure charges

- Immediate publication on acceptance

- Inclusion in PubMed, CAS, Scopus and Google Scholar

- Research which is freely available for redistribution 